\title{
ViewPoint:Gentamicin for treatment of gonococcal urethritis in Malawi
}

\section{Gift Kamanga $^{1}$, Chisale Mhango ${ }^{2}$, Lillian B. Brown ${ }^{3}$}

1.University of North Carolina project, Kamuzu Central Hospital

2.Ministry of Health, Reproductive Health Unit

3..University of North Carolina at Chapel hill

Gonococcal urethritis continues to be a public health problem in Malawi. Urethritis accounts for over $20 \%$ of outpatient STI clinic visits at Kamuzu Central Hospital in Lilongwe annually, and approximately $85 \%$ of urethritis is caused by Neisseria gonorrhoeae ${ }^{1-3}$. Timely and effective treatment of gonococcal urethritis eradicates infection in the affected individual, prevents the development of complications, and reduces the duration of infection, thus preventing onward transmission. Gonorrhea is also an important cofactor for HIV; Neisseria gonorrhoeae infection facilitates HIV transmission, while effective treatment of gonococcal urethritis reduces HIV shedding in the genital tract ${ }^{4}$. Additionally, acute HIV is important to HIV transmission ${ }^{5}$ and the Acute HIV Programme in Malawi found an association between urethritis and likelihood of acute $\mathrm{HIV}^{6}$. Therefore, attention to urethritis is of great public health importance for HIV prevention.

Internationally, several antimicrobial surveillance projects exist to monitor the development and spread of resistance. Two major programs are the Gonococcal Antimicrobial Susceptibility Program (GASP) monitored by the World Health Organization, and the Gonococcal Isolate Surveillance Project (GISP) monitored by the US Centers for Disease Control and Prevention. These programs use laboratory methods to monitor in vitro gonococcal susceptibility and provide an objective measure of resistance. Clinical cure rates, which can be affected by reinfection, are used less often for resistance surveillance.

Malawi adopted syndromic management of sexually transmitted infections in 1993. Based on clinical efficacy and cost, gentamicin 240mg IM and doxycycline $100 \mathrm{mg}$ BID $\times 7$ days was selected as the first line regimen to treat urethritis in the syndromic format ${ }^{7}$. Malawi continues to stand by its recommended use despite WHO and regional recommendations to use ciprofloxacin as first line treatment of uncomplicated gonococcal urethritis. Local surveillance data supports Malawi's decision. A recent article in the journal Sexually Transmitted Diseases showed 100\% susceptibility to gentamicin among men treated for urethritis in the outpatient sexually transmitted infections and reproductive health clinic at Kamuzu Central Hospital in Lilongwe ${ }^{1}$. In this article agar dilution minimum inhibitory concentration (MIC) testing conducted in the United States was used as the gold standard in parallel with less technologically challenging susceptibility methods of disc diffusion and e-test conducted here in Malawi. Results obtained from both MIC testing and the disc diffusion and E-tests performed locally were in agreement for the susceptible isolates. Trials conducted through a collaboration between the $\mathrm{MOH}$ and the University of North Carolina that determined clinical cure rates and/or laboratory based susceptibility data for men with gonoccocal urethritis in Malawi (in both Blantyre and Lilongwe) conducted in 1993, 1996, 1998, and 2001-2, and most recently in 2007 , demonstrate continued susceptibility of gonorrhea to gentamicin at the $>95 \%$ rate $1,3,7$. This continued high susceptibility to the first line drug regimen

stands in contrast to other regions of the world and countries in southern Africa that implemented ciprofloxacin as first line treatment. South Africa saw the proportion of gonococcal isolates resistant to ciprofloxacin increase from zero to over $40 \%$ within 18 months after it was introduced as first line treatment for gonococcal urethritis, 8.9 This dramatic reduction in susceptibility necessitated a switch to more costly drugs, such as cephalosporins. Although the 2007 GC susceptibility survey shows ciprofloxacin to continue to be susceptible in Malawi, it can be expected that resistance would rapidly develop if its use to treat urethritis were increased.

Cost-effective control of gonococcal urethritis is essential in Malawi, particularly given its synergy with HIV. We believe that the Malawi Ministry of Health made the right choice to continue to recommend gentamicin for first line treatment. Toxicity related to gentamicin is related to cumulative doses, and there are no reported toxicities to a single intramuscular injection. However, this has not been looked at systematically and more data are needed on the safety profile of and proper dosing of gentamicin for treatment of gonococcal urethritis. Additionally, as antimicrobial susceptibilities of prevailing gonococcal strains can change rapidly due to the nature of the bacteria, accurate and periodic assessments of susceptibility patterns are needed. Sustaining an accredited microbiology laboratory is challenging, hence previously the majority of the gonococcal susceptibility testing has been performed in external labs outside of Malawi. It's encouraging to note that the e-test and disc diffusion testing were successfully conducted in Malawi and further refinement of these techniques will reduce the dependency on costly testing abroad. While the gold standard for gonococcal microbial sensitivity monitoring is Minimum Inhibitory Concentration (MIC), our study demonstrated high level of agreement between less technically demanding and less expensive tests such as disk diffusion and E-test with the gold standard for susceptible isolates. The use of a turbidometer to standardize turbidity of the culture suspension on the media likely improved the correlation between methods compared to previous susceptibility surveys ${ }^{2}$. This is important because it shows feasibility of microbial susceptibility monitoring in limited resource setting like Malawi. Continued surveillance is necessary to ensure treatment guidelines are in line with current susceptibility trends. Sustaining the local laboratory support will be crucial to these efforts.

\section{References}

1.Brown L, Krysiak R, Kamanga G, et al. Neisseria gonorrhoeae antimicrobial susceptibility in Lilongwe, 2007. Sex Transm Dis 2009 December 2009;36(12).

2.Daly CC, Hoffman I, Hobbs M, et al. Development of an antimicrobial susceptibility surveillance system for Neisseria gonorrhoeae in Malawi: comparison of methods. J Clin Microbiol 1997 Nov;35(11):2985-8.

3.Price MA, Zimba D, Hoffman IF, et al. Addition of treatment for trichomoniasis to syndromic management of urethritis in Malawi: a randomized clinical trial. Sex Transm Dis 2003 Jun;30(6):516-22 
4.Cohen MS, Hoffman IF, Royce RA, et al. Reduction of concentration of HIV-1 in semen after treatment of urethritis: implications for prevention of sexual transmission of HIV-1. AIDSCAP Malawi Research Group. Lancet 1997 Jun 28;349(9069):1868-73.

5.Wawer MJ GR, Sewankambo NK, Serwadda D, Li X, Laeyendecker O, et al. . Rates of HIV-1 Transmission per coital act, by stage of HIV-1 infection, in Rakai, Uganda. J Infect Dis 2005;191:1403-9.

6.Kamanga G, Kwaitana D, Nyirenda N, et al. Identifying Individuals with Acute HIV Who Present to an STI Clinic in Lilongwe, Malawi. XVII International AIDS Coference. Mexico City, 2008:Abstract no THPE0057
7.Lule G, Behets FM, Hoffman IF, et al. STD/HIV control in Malawi and the search for affordable and effective urethritis therapy: a first field evaluation. Genitourin Med 1994 Dec;70(6):384-8.8.Lewis DA, Scott L, Slabbert M, et al. Escalation in the relative prevalence of ciprofloxacin-resistant gonorrhoea among men with urethral discharge in two South African cities: association with HIV seropositivity. Sex Transm Infect 2008 Oct;84(5):352-5.

9.Moodley P, Sturm AW. Ciprofloxacin-resistant gonorrhoea in South Africa. Lancet 2005 Oct 1;366(9492):1159.

National Commission for Science and Technology (NCST), Health Research Capacity Strengthening Initiative (HRCSI). 1st Floor Lingadzi House .P/B B303, City Centre, Lilongwe, Malawi.

\section{Funding Opportunities from HRCSI}

The National Commission for Science and Technology (NCST) is implementing a five-year Health Research Capacity Strengthening Initiative (HRCSI). HRCSI is funded by the UK Department for International Development (DFID), Wellcome Trust and International Development Research Centre (IDRC). The overall goal of HRCSI is to build and strengthen health research capacity at both individual and organisational levels in Malawi. At individual level, it provides training and research grant fellowships. At institutional level, HRCSI supports the development of research infrastructure.

HRCSI offers scholarships to Malawians at Masters and PhD levels. Fellowships target particular disciplines. At Master's level, the following are target disciplines: (a) Biostatistics (b) Demography (c) Health economics (d) Medical anthropology (e) Medical biology (f) Medical microbiology (g) Medical pathology (h) Medical chemistry (i) Epidemiology and (j) Health systems: health care financing, human resources for health, health information systems, health management and organization, health programme evaluation, procurement and supply chain management and health policy. At PhD level, HRCSI scholarships support applicants who intend to specialise in any of the following disciplines: (a) Biostatistics (b) Health Economics (c) Medical Microbiology and (d) Medical pathology. HRCSI does not process any application from an applicant who intends to specialise in any other field apart from the ones listed above.

HRCSI makes competitive grants to researchers. There are three types of research grants. These are postgraduate dissertation research grants, junior and senior research grants. Postgraduate Dissertation Research Grants seek to facilitate more rigorous engagement of postgraduate students in research. The maximum values of Masters and $\mathrm{PhD}$ dissertation research grants are $\$ 4,000$ and $\$ 10,000$, respectively.

To qualify for postgraduate research grants, applicants must (a) be Malawian citizens (b) be pursuing a Masters or PhD training at a recognised university (c) have completed all pre-dissertation requirements e.g. coursework (d) have a research dissertation proposal that s on health or health-related topic and (e) provide evidence that the research proposal has been approved by their supervisors.

Junior research grants support novice researchers who want to develop their career in health research. The value of each junior research grant is a maximum of US\$20,000 per year for up to three years. The applicant for junior research grants must: (a) be a Malawian citizen affiliated with a Malawian institution and (b) and have a health-related research topic relevant to Malawi.

Senior research grant is offered to researchers with a proven track record of conducting research and managing research grants. The value of senior research grant is a maximum of US $\$ 70,000$ per year for up to three years. Applicants must: (a) be Malawian citizens affiliated with a Malawian institution (b) have at least three publications in international journals as the first author in the past five years

(c) have a team of researchers that includes at least one junior researcher with at least a master's degree and (d) have at least one student who will graduate with a Masters or $\mathrm{PhD}$ degree at the end of the project.

The application process for training fellowship is different from that of the research grants. For training fellowships, applicants can only make applications to HRCSI after HRCSI has made request for submission of applications. Calls for training fellowship applications are made twice in a year. Submission of applications for research grants is open. There is no restriction on time when applicants can submit their research proposals to HRCSI. That is, applicants can submit their applications throughout the year.

The review of research grant application proceeds in three stages. These are in-house screening, external and MRC review. In-house screening seeks to eliminate weaker proposals from the pool of proposals. This allows subsequent reviews to focus on proposals are most likely to be funded. Proposals shortlisted in the in-house screening are sent to three external reviewers. External reviewers are independent experts who assess the scientific merit of the proposal. The Multidisciplinary Research Committee (MRC) comprises independent scientists who adjudicate on views of external reviewers. The MRC meets four times in a year to review research grant applications. The earlier in the quarter an application is submitted the higher the likelihood that it will be reviewed within that quarter. HRCSI communicates with applicants the outcome of the results of the review process within one month of the MRC meeting. For more information and further details visit HRCSI website at: http://www.hrcsi.ncst.mw or email: hrcsi@ncst.mw. 